\title{
Revisión
}

\section{Incidencia del cáncer a nivel mundial. Revisión sistemática.}

Worldwide cancer incidence. A systematic review

\author{
Carmen Bernabeu Aguirrer ', Davinia Gomis Juan ${ }^{* 2}$, \\ Estefanía lsabel Martínez Canals', Jorge Bernabeu Aguirre ${ }^{3}$ \\ ${ }^{1}$ Hospital General Universitario de Alicante, ${ }^{2}$ Hospital General Universitario Santa \\ María del Rosell en Cartagena ${ }^{3}$ Imed Torrellano.
}

*Correspondencia daviniagomis@hotmail.com

\section{Resumen}

\section{Introducción:}

La población mundial está en continuo crecimiento conllevando un incremento de los casos.

El desarrollo social y económico influye en el perfil del cáncer. Conforme se prospera y los ingresos aumentan, el cáncer crece constante $y$ de manera uniforme. (2)

Según datos oficiales de la Organización Mundial de la Salud (OMS), en las próximas dos décadas el número de casos de cáncer podría aumentar hasta en un $60 \%$, registrándose hasta un $80 \%$ en los países de bajo y medio ingreso.

\section{Objetivos:}

Conocer la tendencia actual del Cáncer y los factores de riesgo relacionados con su aparición y avances al respecto.

\section{Método:}

Revisión sistemática de la literatura científica en bases de datos de interés científico nacional e internacional. Se emplea la revisión narrativa basada en la interpretación del lenguaje. Se emplea la reflexión para la comprensión de los resultados.

\section{Resultados:}

El cáncer de pulmón es el más letal de entre todos. Los factores de riesgo más influyentes son el abuso de alcohol y tabaco, sedentarismo, mala alimentación desencadenando un mal pronóstico.

Se aprecian numerosos estudios, novedosos y actuales. El avance de la investigación es lento, aunque progresivo. 
Australia es el país que registra la mayor tasa de incidencia de cáncer y la primera que invirtió en pruebas diagnósticas y protocolos de actuación.

\section{Conclusión:}

El cambio que la sociedad está experimentando implica un incremento y avance de las neoplasias debido a la occidentalización, ya que la adquisición y puesta en práctica de factores de riesgo los ponen en el punto de mira, perjudicando su salud.

Se observa consigo la relación existente entre el nivel adquisitivo del territorio, comprobando que, a mayor Índice de Desarrollo Humano (IDH), mayor incidencia y a su vez mas inversión en investigación.

Palabras clave: Neoplasia, morbilidad, mundial, riesgo a la salud.

\section{Abstract}

\section{Introduction:}

Worldwide population is always immersed in a constant growing process that leads to a remarkable increase in cancer cases.

It is said that socioeconomic development has a certain influence in cancer profile. As people social conditions improve and economic income increases, cancer disease grows constantly and uniformly.

According to the official datum given by the World Health Organization (WHO), the number of cancer cases could increase in the following couple of decades. This increasement could reach numbers about $60 \%$, and in low- income and middle-income countries about $80 \%$.

\section{Objectives:}

To know the present cancer incidence in the world, and the risk factors related to the disease appearance and development.

\section{Method:}

Systematic revision of the scientific literature given by the relevant national and international scientific data bases. The present research is focused on a narrative revision based on a language analysis and a data reflection that leads to the comprehension of the research results.

\section{Results:}

Lung cancer is the most lethal type of cancer.

The most influential risk causes are alcohol and tobacco abuse, sedentariness, and deficient nutrition. They all lead to a negative prognosis.

There is a great number of contemporary and innovative scientific research.

Cancer research development is slow but progressive.

The highest cancer incidence rate in a country is registered in Australia. It was the first country that invested in diagnostic testing and action protocols.

\section{Conclusion:}

The change that worldwide society is suffering implies the increment and development of neoplasia due to a westernization process. This issue causes the acquisition and implementation of cancer risk factors that put people in the 
spotlight and make their health get worse.

It can be observed that there is a relation between an area purchasing power and the number of cancer cases. Thus, it has been confirmed that the more the Human Development Index (HDI) is the more incident and, at the same time, the more investment in research there is.

Keywords: Neoplasia, morbidity, Global Health, health risk.

\section{Introducción}

El cáncer o tumor maligno, ocasiona un proceso descontrolado en la división celular del organismo (anormalidades en el material genético de las células). (1)

Puede comenzar de manera localizada y diseminarse por los tejidos circundantes pudiendo llegar a propagarse por otros órganos (metástasis) conduciendo a la muerte, si el paciente no recibe el tratamiento adecuado. (1)

La población
mundial está en
continuo
crecimiento
envejece
rápidamente debido a los cambios en la fertilidad $y$ esperanza de vida, produciendo un aumento de los casos. (2)

Con la evolución a niveles más altos del desarrollo, el comportamiento general de la población también aumenta. El desarrollo social y económico influye en el perfil del cáncer, que conforme los países prosperan y los ingresos aumentan se observa un crecimiento constante $y$ uniforme de éste. (2)

Algunos países de Índice de Desarrollo Humano (IDH) muy alto son Australia, Alemania, Nueva Zelanda, España, Suecia, Reino Unido, Estados Unidos, con IDH alto nos encontramos con Argelia, China, México, Rusia, con IDH medio Bolivia, Egipto, Maldivas, Marruecos e IDH bajo Afganistán, Camboya, Nigeria, Senegal entre otros. (2)

Debido al incremento progresivo de las tasas a nivel mundial supone un reto importante llevar a cabo la prevención, aun suponiendo un desafío de salud pública que hoy en día cuesta lograr un resultado adecuado ${ }^{(1)}$.
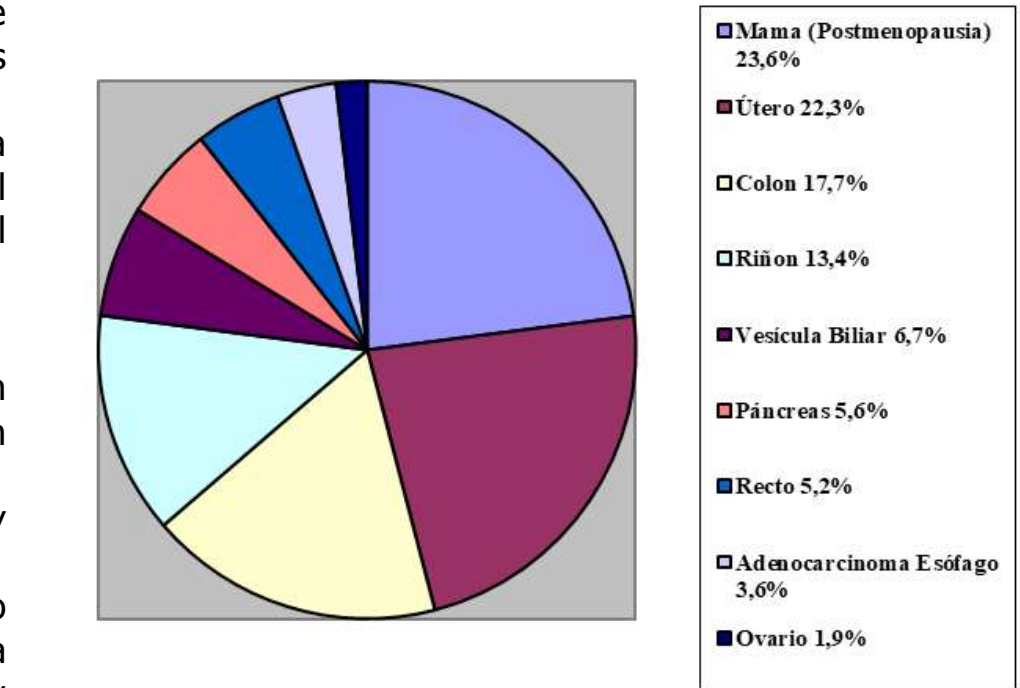

Figura 1.- Porcentajes atribuibles a la obesidad a nivel mundial para el año 2012, por localización tumoral. (Creación propia)

Según datos oficiales de la Organización Mundial de la Salud (OMS), en las próximas dos décadas el número de casos de cáncer podría aumentar hasta en un $60 \%$, registrándose hasta un 
$80 \%$ en los países de bajo y medio ingreso ${ }^{(1)}$

El cáncer es un factor importante de la carga mundial de morbilidad (1) y una de las causas principales de muerte alrededor del mundo. Los cambios en la estructura de la población influyen en las proyecciones de casos de cáncer en el futuro. (5)

Existe la necesidad (Según la OMS) de aumentar los servicios de tratamiento del cáncer en aquellos países que cuenten con ingresos medios y bajos debido al futuro aumento de casos. (28)

En el informe redactado en febrero de 2020 se indica que una de cada 6 personas morirá de cáncer cada año y que la carga de esta patología aumentará, registrándose un número total de muertes por cáncer superior a 11,5 millones de fallecimientos en 2030 y alrededor de los 29,5 millones en el año $2040^{(1)}$.

Esto conllevará el colapso de los sistemas de salud (4).

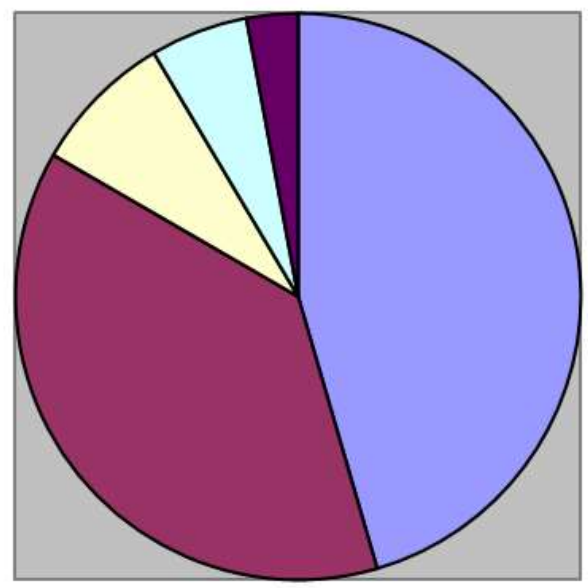

Figura 2.-Porcentaje de casos tumorales atribuibles a la exposición a radiación ultravioleta, por continente (Elaboración Propia)
Dicho desafío debe implicar una acción y puesta en común de todos los ámbitos y sectores de la sociedad, fortaleciendo las acciones desde edades tempranas $y$ desde el seno familiar, instituciones educativas y la sociedad (29)

Los gobiernos deben desempeñar un papel fundamental para impulsar cambios en el comportamiento de las personas sobre alimentación y actividad física. (29)

Por ello en mayo de 2004 se llevó a cabo la 57a Asamblea Mundial de la Salud donde se Aprobó la Estrategia Mundial de la Organización Mundial de la Salud sobre Régimen Alimentario, Actividad Física y Salud. (29)

Datos que registran dicha estrategia, confirman que la alimentación y actividad física aportan beneficios a la salud, mejoran la salud física y mental e implicaría una reducción de la morbilidad mortalidad. (29)

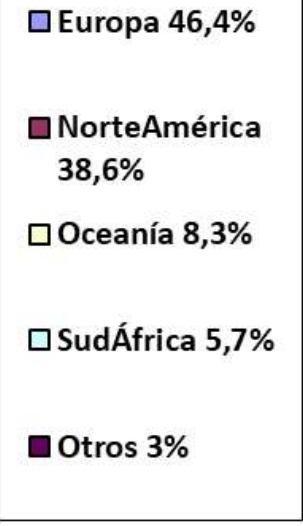

Datos del American Institute for Cáncer, países como Australia registra la tasa más elevada de cáncer con 468 personas por cada 100.000 y en global las mayores cifras se encuentran entre Oceanía, Europa y América del norte. 


\begin{tabular}{|c|}
\hline 口Pulmón $11,6 \%$ \\
\hline 口Mama $11,6 \%$ \\
\hline 口Colorrectal $10,2 \%$ \\
\hline 口Próstata 7,1\% \\
\hline Estómago 5,7\% \\
\hline 口Hígado 4,7\% \\
\hline DEsófago 3,2\% \\
\hline 口Cérvix 3,2\% \\
\hline Tiroides $3,1 \%$ \\
\hline 口Vejiga 3\% \\
\hline $\begin{array}{l}\text { ㄴinfoma no Hodgkin } \\
2,8 \%\end{array}$ \\
\hline 口Páncreas 2,5\% \\
\hline 口Leucemia 2,4\% \\
\hline 口iñón 2,2\% \\
\hline 口Útero 2,1\% \\
\hline DOtros $24,6 \%$ \\
\hline
\end{tabular}

seguidos del cáncer mama $(11,6 \%)$, colorrectal $(10,2 \%)$ y próstata $(7,15 \%)$.

El consumo de tabaco influye en el desarrollo del cáncer de pulmón, implicando un $20 \%$ de muertes. Si la tendencia se mantiene éste llegará a producir la muerte de

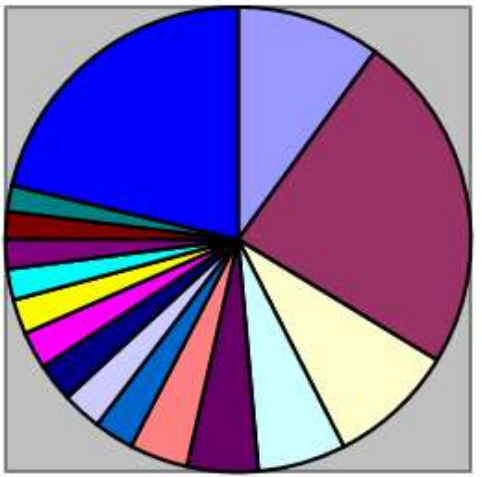

7 millones de personas en 2020 y 8 millones para el 2030. (4)

En España, según el informe anual de la Sociedad Española De Oncología Médica (SEOM) se registran un total de 277.394 casos, observando un incremento en mujeres de casi un $1 \%$ respecto a 2019 y un descenso de 0,5 en hombres. ${ }^{(4)}$

De acuerdo con el Instituto Nacional de Estadística (INE), los tumores constituyen la segunda causa de muerte en España por detrás de patologías del sistema circulatorio. (4)

Entre los varones en España se puede indicar

Figura 3.- Estimación para el 2018 en los tumores más frecuentes diagnosticados a nivel mundial, ambos sexos. (Creación propia)

Se observa que el cáncer de pulmón es uno de los mayores y más peligrosos enemigos de la humanidad, resultando ser más frecuente y causante de la mayor mortalidad por neoplasias malignas a escala mundial, registrando una muerte por cada 30 segundos en el mundo (1), que los tumores fueron la primera causa de muerte y la segunda en mujeres, registrando las mayores tasas de mortalidad en zonas como el Principado de Asturias, Extremadura y Melilla. (4)

Según datos estadísticos del gobierno australiano estima alrededor de 150.000 casos nuevos diagnosticados y sobre las 50.000 defunciones por cáncer, donde el de mama es el cáncer 
diagnosticado con mayor frecuencia seguido del de próstata y observando un descenso del cáncer de pulmón desde el año 2000 pero, aun así, éste, sigue siendo la principal causa de muerte.

Este número tan elevado de casos conlleva un gran impacto social y económico tanto a nivel individual, familiar y comunitario.

En Europa, países como Alemania la cuarta parte de los hombres y el $20 \%$ de las mujeres fallecen por cáncer $(51 \%$ de hombres y $43 \%$ de mujeres lo desarrollaran durante su vida). (5)

Para el 2030 se estima que el cáncer de próstata será la neoplasia más común superando al de mama y el de pulmón pasará a ocupar el tercer puesto de los más frecuentes y seguirá siendo la causa más común de mortalidad. (5)

Se observa un incremento del cáncer de páncreas y de mama, donde se convertirá en la segunda causa de muerte más común en Alemania para 2030. (5)

\section{Objetivo general}

Conocer la tendencia actual del Cáncer y los factores de riesgo relacionados con su aparición y avances al respecto.

\section{Objetivo especifico}

- Identificar el cáncer más letal actualmente a nivel mundial, así como el país con mayor incidencia.

- Determinar los factores que influyen en la aparición y/o predisposición al desarrollo de masas tumorales.

\section{Hipótesis}

El alto índice de mortalidad relacionada con el cáncer está asociado a los factores de riesgo.

Reforzar las intervenciones individuales y sociales, interaccionando con el paciente e implicando a los medios sanitarios y sociales contribuirían a la concienciación.

Actividades preventivas y de cambio de comportamiento determinan una mejora en la calidad de vida, así como el nivel de autocuidado, competencias, control de complicaciones, aportando conocimientos y resultando útiles para todos.

\section{Método}

Revisión Sistemática de la Literatura Científica, utilizando la revisión narrativa. Es útil para el análisis de investigaciones basada en la interpretación del lenguaje. Mediante un enfoque inductivo y utilizando la reflexión para la comprensión de los resultados, facilita la introducción de múltiples visiones, creando así una mezcla de significados (6). Respecto a los tipos de síntesis de la evidencia descrito por Noblit y Hare (1988) y revisados por Dixon-Wood y colaboradores ${ }^{(7)}$, cabe señalar que esta revisión se incluye dentro de la síntesis interpretativa de los datos.

\section{Bases de datos}

Las investigaciones llevadas a cabo se centran en una búsqueda exhaustiva de la literatura relacionada con el cáncer, la tendencia actual, factores de riesgo y los avances científicos al respecto, realizándose a través de bases de datos del mundo de la 
salud que resultan fiables $y$ reconocidas.

Las búsquedas se realizaron en las siguientes bases de datos: Cochrane, Scielo, y Medline.

Las palabras clave o descriptores utilizados para la búsqueda científica han sido (Tabla 1 ):

- Español: Neoplasia; indicadores de salud, Europa; incidencia, mortalidad, en todo el mundo, oncología médica, morbilidad, riesgo a la salud.

-Inglés: Neoplasia; health indicators, Europe; incidence, mortality, Global Health, medical oncology, health risk.

Las palabras clave indexadas corresponden a Descriptores en Ciencias de la Salud (DeCS).
Se indica que no existe una equivalencia del $100 \%$ entre los DeCS y los MeSH, siendo estos últimos los usados por la base de datos Medline. Existe, por tanto, algunos problemas en cuanto a la reproducibilidad de la búsqueda. Siendo un ejemplo de ello el descriptor de salud, Worlwide, que los autores han utilizado en la búsqueda en Medline y que no se corresponde con un MeSH reconocido

Para la realización de la búsqueda se han combinado las palabras clave mediante el operador booleano AND.

El año de inicio de Búsqueda se realiza a partir de 2015 realizando ésta tanto en español como en inglés, y que su acceso sea "free

\section{Tabla 1}

Descriptores de salud empleados y equiparaciones entre términos.

\begin{tabular}{|l|l|}
\hline DeCS & MeSH \\
\hline Neoplasia & Breast Neoplasms \\
\hline Indicadores De Salud & No Localizado \\
\hline Europa & Europe \\
\hline Incidencia & Epidemiology \\
\hline Mortalidad & Mortality \\
\hline Worldwide & Global Health \\
\hline Oncología Médica & Medical Oncology \\
\hline Morbilidad & Morbidity \\
\hline Riesgo A La Salud & Health Risk \\
\hline
\end{tabular}

Tabla de elaboración propia.

Indicar que entre los DeCS y los $\mathrm{MeSH}$ no existe una equivalencia del $100 \%$, siendo los MeSH aquellos empleados por bases de datos como MedLine. Por tanto, existen ciertos problemas en cuanto a la reproductibilidad de la búsqueda. full text" así como que sean Metaanálisis, ensayo controlado aleatorio, revisiones y revisiones sistemáticas. 


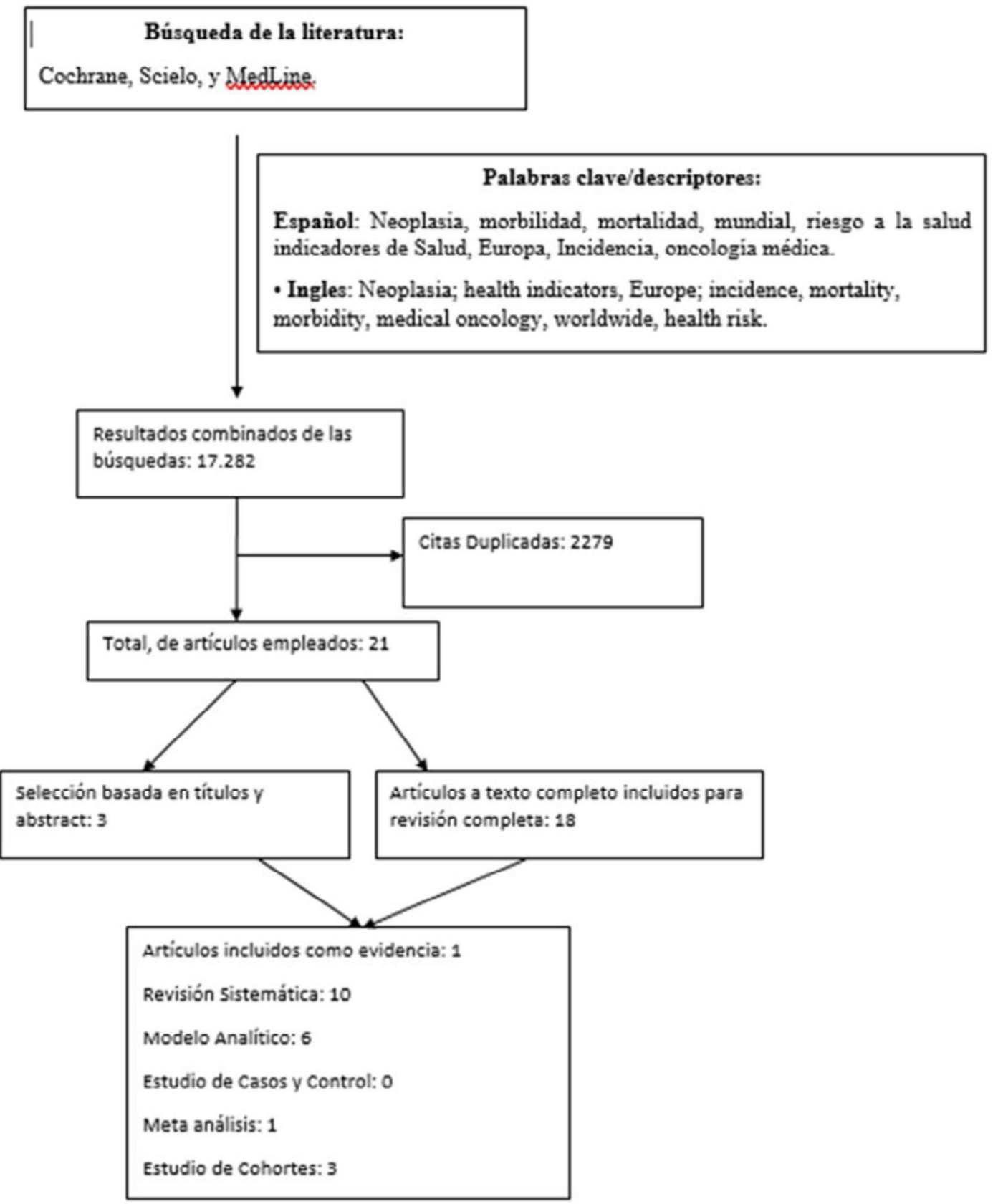

Figura 4 Diagrama de flujo de la revisión. 
Con las palabras clave que a continuación se detallan y en las bases de datos que se pueden observar en las tablas adjuntas, no se selecciona ningún artículo que no esté dentro de las características necesarias para formar parte de nuestro trabajo.

\section{Ecuaciones de búsqueda:}

Palabra clave $1^{0}$ búsqueda:

- Neoplasia and Indicadores de salud and Europa and Incidencia and Mortalidad

Se localizan 0 artículos en Cochrane $\AA, 0$ en Scielo $\AA$ y 0 en

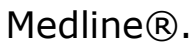

- Neoplasia and health indicators and Europe and incidence and mortality

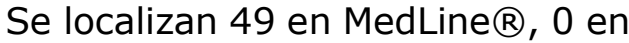

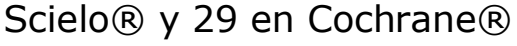

Palabra clave 20 búsqueda:

- Neoplasia and Indicadores de salud and Incidencia and Mortalidad

Se localiza 0 artículos en Medline $\AA$, 1 en Scielo $\AA$, 0 en Cochrane $\mathbb{R}$

- Neoplasia AND health indicators and incidence and mortality

Se localiza 1 en Cochrane $\AA$, 0 en

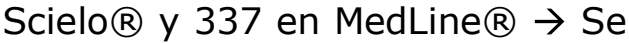
emplean 2 de MedLine $\circledR$.

Palabra clave 30 búsqueda:

-Neoplasia AND mundo AND mortalidad

Se localiza artículos en 0 Medline $\AA$, 26 en Scielo $\AA$, 0 en Cochrane $\circledR$
-Neoplasia AND Global Health AND mortality:

10 en Scielo $\AA$, 0 en Cochran $\circledast$ e y 249 en MedLine $\mathbb{R} \rightarrow$ Se emplean 2

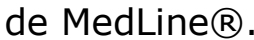

Palabra clave $4^{\circ}$ búsqueda:

-Neoplasia and incidencia

Se localizan 0 en Cochran $囚 e, 507$

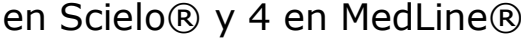

-Neoplasia AND incidence

Se localizan 12.705 en Medline $\AA$ y 515 en Scielo ${ }^{\circledR}$ y 16 en

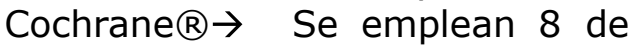
MedLine $\AA$

Palabra clave $5^{a}$ búsqueda:

- Neoplasia AND indicadores de salud and Europa

Se localiza artículos en 0 Medline $\AA$, 0 en Scielo $\AA, 0$ en Cochrane $\circledR$

- Neoplasia AND health indicators and Europa

Se localiza 1 artículo en MedLine ${ }^{\circledR}$,

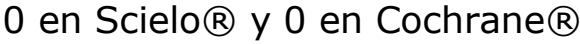

Palabra clave $6^{a}$ búsqueda:

- Neoplasia AND incidencia AND mortalidad and oncología médica

Se localiza artículos en 1 Medline ${ }^{\circledR}, 0$ en $S c i e l o \circledR, 0$ en Cochrane $\mathbb{R}$

-Neoplasia AND incidence AND mortality AND medical oncology

Se localizan 2058 artículos en MedLine $\AA$, 1 en Scielo $\AA$ y 0 en Cochrane $\mathbb{R} \rightarrow$ Se emplean 2 de MedLine $®$. 
Palabra clave 7 a búsqueda:

-Neoplasia AND incidencia and mundo.

Se localiza artículos en 0 Medline $\AA$, 46 en Scielo $\AA$, 0 en Cochrane $\AA$

-Neoplasia AND incidence AND Global Health

Se localiza artículos en 573 Medline ${ }^{\circledR}, 7$ en Scielo $\AA$, 1 en Cochrane $\AA \rightarrow$ Se emplean 3 de MedLine $\circledast$.

Palabra clave $8^{a}$ búsqueda:

-Neoplasia AND morbilidad AND mundial AND riesgo a la salud

Se localiza artículos en 0 Medline $\AA$, 0 en Scielo $\AA$, 0 en Cochrane ${ }^{\circledR}$

-Neoplasia AND morbidity AND Global Health and health risk

Se localizan 0 en Cochrane $\AA$, 1 en Scielo $\AA$ y 287 en MedLine $\AA \rightarrow$ Se emplean 3 de MedLine ${ }^{\circledR}$.

Palabra clave 9 búsqueda:

-Neoplasia AND mundial AND riesgo a la salud

Se localiza artículos en 0 Medline $\AA$, 12 en Scielo $\AA, 0$ en Cochrane $\AA$

-Neoplasia AND Global Health AND health risk

Se localizan 1 en Cochrane $\AA, 13$ en Scielo $\AA$ y 389 en MedLine $\AA$ $\rightarrow$ Se emplean 1 de MedLine ${ }^{\circledR}$.

\section{Criterios de inclusión y exclusión.}

\begin{tabular}{|c|c|}
\hline $\begin{array}{l}\text { Criterios } \\
\text { Inclusión. }\end{array}$ & $\begin{array}{l}\text { Criterios } \\
\text { Exclusión. }\end{array}$ \\
\hline $\begin{array}{l}\text { Estudios, artículos, } \\
\text { tesis y ponencias } \\
\text { que } \\
\text { relación con en el } \\
\text { cáncer a nivel } \\
\text { mundial. }\end{array}$ & $\begin{array}{l}\text { Estudios, } \\
\text { artículos, tesis y } \\
\text { ponencias que } \\
\text { NO tengan } \\
\text { relación con el } \\
\text { cáncer a nivel } \\
\text { mundial. }\end{array}$ \\
\hline $\begin{array}{l}\text { Estudios, artículos, } \\
\text { tesis y ponencias } \\
\text { que traten del } \\
\text { cáncer, incidencia } \\
\text { y evolución. }\end{array}$ & $\begin{array}{l}\text { Estudios, } \\
\text { artículos, tesis y } \\
\text { ponencias } \\
\text { que NO traten del } \\
\text { cáncer, } \\
\text { incidencia } \\
\text { evolución. y }\end{array}$ \\
\hline $\begin{array}{l}\text { Estudios, artículos, } \\
\text { tesis y ponencias } \\
\text { que traten del } \\
\text { cáncer y los } \\
\text { factores de riesgo } \\
\text { para la salud de la } \\
\text { población. }\end{array}$ & $\begin{array}{l}\text { Estudios, } \\
\text { artículos, tesis y } \\
\text { ponencias } \\
\text { Que NO traten } \\
\text { del cáncer y los } \\
\text { factores } \text { de } \\
\text { riesgo para la } \\
\text { salud de la } \\
\text { población. }\end{array}$ \\
\hline $\begin{array}{lr}\text { Aquellos que por } \\
\text { su pertenencia, } \\
\text { relevancia ra } \\
\text { actualidad son } \\
\text { interesantes para } \\
\text { el desarrollo del } \\
\text { Trabajo. }\end{array}$ & $\begin{array}{l}\text { Aquellos que tras } \\
\text { la lectura de sus } \\
\text { abstract no se } \\
\text { ajustan a nuestro } \\
\text { objeto de estudio } \\
\text { o presentan } \\
\text { niveles de } \\
\text { evidencia bajos. }\end{array}$ \\
\hline
\end{tabular}

Tabla 2. Criterios se inclusión y exclusión.

\section{Resultados}

\section{Descripción de los resultados encontrados.}

Se identifican unas categorías principales, que agrupan los conceptos que emergen de la lectura y el análisis del contenido de las publicaciones seleccionadas. 
El cáncer en el mundo: Los artículos que a continuación se detallan coinciden en identificar como el cáncer más letal el de pulmón ${ }^{(5)}$, asociado a su vez a factores de riesgo como el consumo de tabaco como factor predisponente a su aparición, seguidos del colorrectal, mama, hígado y estómago. Se puede apreciar como las mayores tasas se producen en los países más desarrollados ya que en parte el estilo de vida de la sociedad tiende a incrementar su aparición, por tanto, llevar un estilo de vida plagado de factores de riesgo pone en peligro la vida de los individuos. (13) (20)

Por otro lado, los países con índices más desarrollados invierten más en tecnología, estudios y avances para paliar y mitigar este avance. (13) Observando como en aquellos países más pobres y con menos opciones de diagnóstico y avance, cada vez más las tasas de aparición del cáncer están en incremento y con ello el índice de mortalidad. (13)

Calidad de vida y Factores de riesgo: Una vida sedentaria en el que la realización de ejercicio no forma parte del día a día del individuo, el abuso en el consumo de tabaco y alcohol, una mala alimentación con escaso consumo de frutas y verduras influyen en una mala calidad de vida y en incrementar las probabilidades de que se desarrolle un tumor a cualquier nivel del organismo, por ello resulta fundamental una adecuación por parte del individuo y concienciación de la sociedad para cambiar los hábitos. (29)
En aquellos territorios con mayor índice de desarrollo existen porcentajes elevados de casos de cáncer y a su vez también se observa una creciente curva de individuos que están realizando cambios en su estilo de vida, están adecuando su rutina cotidiana a realizar ejercicio (15) (21), cambiar sus hábitos alimenticios y reducción del consumo de alcohol y tabaco. (13)

A pesar de todo aún queda mucho camino por realizar.

Avances: Se aprecia claramente, sobre todo en la fecha de publicación de los artículos, que cada día se está investigando al respecto, poniendo en práctica los estudios y los avances y sus resultados, (14) (16) aunque se debe investigar más para seguir progresando ya que queda camino por recorrer y por avanzar para ir frenando el desarrollo de tumores.

\section{Sistematización de los trabajos revisados.}

En la tabla se presenta el resumen de los artículos seleccionados, incluyendo autor, año, objetivo del estudio, tipo de estudio y elementos útiles.

Se exponen un total de veintiún artículos seleccionados, después de cumplir los requisitos establecidos.

\section{Tabla 3}

\section{Conclusiones}

Se aprecia un cambio en la sociedad, una transición entre las neoplasias relacionadas con infecciones hacia las neoplasias debido a la occidentalización, debido al incremento de la adquisición y puesta en práctica de 
factores de riesgo perjudiciales para la salud.

Se puede apreciar en el cáncer colorrectal, incrementándose entre 6 y 7 veces más en regiones de Índice de Desarrollo Humano (IDH) muy alto, todo ello ligado al estilo de vida y medio ambiente junto factores dietéticos $y$ metabólicos. (1) (11) (27)

En contrapartida se observa que según el IDH, la investigación en el campo de la oncología, también varia, con un incremento sustancial en países de IDH elevado, (13) (20) ejemplo de ello es la detección mamográfica para el cáncer de mama, que a pesar de seguir siendo un desafío, la reducción de la incidencia en países desarrollados atraviesa una transición importante ya que los esfuerzos para disminuir la obesidad y el consumo de alcohol y fomentar la lactancia materna pueden tener un impacto en su reducción. ${ }^{(28)}$

Políticas y reglas eficaces para el control del tabaco implicaría una reducción de la carga de cáncer de pulmón. Actualmente existen herramientas como el Convenio Marco para el Control del Tabaco de la Organización Mundial de la Salud y las medidas MPOWER. (2)

Dichas políticas dependen del país del que hablemos ya que varía la incidencia y mortalidad todo ello relacionado con el desarrollo económico.

A su vez es necesario una concienciación y cambio en el estilo de vida asociados con la occidentalización, claro ejemplo es la elevada tasa de obesidad y /o el consumo de tabaco.
También estrategias de prevención primaria que controlen estos factores, junto programas de vacunación y detección temprana, un diagnóstico preciso y el acceso a la terapia multimodal adecuada. (13) (28)

Como indica la OMS: identifica 4 áreas para el éxito en los programas de control del cáncer: modificación y prevención de factores de riesgo, diagnóstico temprano, tratamiento y paliación. (13) (29).

Si no, en unos años se producirá una sobrecarga de los recursos sanitarios por un crecimiento $y$ envejecimiento continuo de la población, como es el caso de Corea del Sur donde el cáncer es la principal causa de muerte. (18)

La tendencia actual del cáncer es una tendencia al alza que, a pesar de la detección temprana, la prevención, tratamiento y paliación su incremento no cesa, si bien se frena en ciertos países por dichos aspectos, pero es la nueva patología del siglo XXI, donde los factores de riesgo ya descritos son los predisponentes para su aparición. Se puede indicar por ejemplo que, el cáncer se ha convertido en la segunda enfermedad más grave que amenaza la salud humana, seguida de las enfermedades cardiovasculares. (9)

El cáncer sigue siendo un importante desafío de salud pública en todo el mundo, un problema de salud complejo y creciente que no solo debe ser función de la atención médica, sino que requiere del esfuerzo global y generalizado y garantizar que los daños físicos, psicológicos y financieros, se minimicen y que los 
beneficios superen los riesgos agregados. (11) (23)

Actualmente uno de los canceres más letales y más relacionado con los factores de riesgo es el cáncer de pulmón debido al consumo de tabaco, éste, influye en el desarrollo del cáncer de pulmón, implicando un $20 \%$ de muertes. Si la tendencia se mantiene éste llegará a producir la muerte de 7 millones de personas en 2020 y 8 millones para el 2030. (4)

En definitiva, la prevención es la estrategia contra el cáncer a largo plazo más rentable, poco a poco el cáncer se está convirtiendo en una patología crónica que implica mucho tiempo de tratamiento, la puesta en práctica de estrategias como la angiopreventiva comentada anteriormente basadas en los componentes de la dieta mediterránea pueden ayudar a controlar la enfermedad. (14) (16) (19)

Un concepto emergente que está reportando consecuencias importantes y beneficiosas respecto a la prevención del cáncer y otras patologías. (14)

Como indica el doctor Tedros Adhanom Ghebreyesus, Director General de la OMS. "Si las personas tienen acceso a la atención primaria y luego a los sistemas de derivación, es posible detectar temprano el cáncer, tratarlo eficazmente y curarlo. El cáncer no debería ser una pena de muerte para nadie, en ninguna parte del mundo." (28)

Se observa un número importante de artículos que tratan de los avances en la investigación contra el cáncer, desarrollo de fármacos, terapias dirigidas con inhibidores (24), estrategias de prevención como la vacunación contra el VPH (17) , identificación de biomarcadores en sangre (25) , estrategias angiopreventivas como la realización de actividad física (15) (21) y la alimentación para prevenir y controlar la enfermedad (14), están progresando cada día y realizando avances $y$ descubrimientos para poder ayudar a la población para así tener una adecuada calidad de vida. (14) (16) (25)

A pesar de que los artículos incluidos en el estudio son de reciente publicación, se considera una limitación importante la escasa la cantidad de ellos y la falta de una mayor proporción de artículos que se centren en la incidencia y prevalencia del cáncer a nivel mundial y a su vez más ensayos en los que las terapias y estrategias novedosas ante el cáncer y su propagación se pongan en marcha. (19)

Si bien es cierto en los últimos años y coincidiendo con los años de búsqueda en las bases de datos nacionales e internacionales, son numerosos los estudios, pero poco que realicen ensayos con terapias vanguardistas para el avance de su detección y freno.

Países como Australia, España o Estados Unidos presentan un incremento en la supervivencia gracias a actividades preventivas (Australia fue el primer país en implementar la vacuna del VPH (17) , campañas de diagnóstico precoz, avances terapéuticos y disminución del consumo de tabaco. (4) 


\section{Referencias}

1.- Cencomed. Centro virtual de Convenciones de Salud. González -Abreu Báez, A., Morejón Gamboa, L., Falcón Díaz, C., Gamboa Díaz Y. "Caracterización Epidemiológica Del Cáncer.". [Internet]; 2020. Primer Congreso Virtual de Ciencias Básicas Biomédicas en Granma. Manzanillo, Cuba. [Acceso el 17 de agosto de 2020] Disponible en: http://www.cibamanz2020.sld.cu/index.p hp/cibamanz/cibamanz2020/paper/view/ $589 / 351$

2.- Fidler, M. M., Soerjomataram, I., \& Bray, F. "A global view on cancer incidence and national levels of the human development index". [Base de datos de internet] 2016. International journal of cáncer, 139(11), 2436-2446. https://doi.org/10.1002/ijc.30382

[Acceso el 17 de Agosto de 2020] Disponible en: https://onlinelibrary.wiley.com/doi/full/10 .1002/ijc.30382

3.- Bray F, Ferlay J, Soerjomataram I, Siegel RL, Torre LA, Jemal A. "Global Cáncer Statistics 2018: estimaciones de GLOBOCAN de incidencia y mortalidad en todo el mundo para 36 cánceres en 185 países".[Internet] CA Cáncer J Clin, en prensa.[Acceso el 17 de agosto de 2020] Disponible en: http://gco.iarc.fr/

4.- Sociedad Española de Oncología Médica (SEOM), "Las cifras del cáncer en España 2020". [Internet] 2020. [Acceso 17 de agosto de 2020] Disponible en: https://seom.org/seomcms/images/storie s/recursos/Cifras_del_cancer_2020.pdf 5.- Quante, AS, Ming, C., Rottmann, M., Engel, J., Boeck, S., Heinemann, V., Westphalen, CB y Strauch, K. "Proyecciones de incidencia de cáncer y muertes relacionadas con el cáncer en Alemania para 2020 y 2030". [base de datos de internet] 2016. Medicina contra el cáncer, 5 (9), 2649-2656. [Acceso el 17 de agosto de 2020] https://doi.org/10.1002/cam4.767

Disponible en: https://www.ncbi.nlm.nih.gov/pmc/article s/PMC5055190/

6.- Greenlund, K.J., y Croft, J.B. (2000). The poetic imension. Therapeutic potentials. En K. Desissler y S.M. Namee 7.- Dixon-Woods, M., Agarwal, S., Jones, D., Young, B., y Sutton, A. (2005).
Synthesising qualitative and quantitative evidence: a review of possible methods. Journal of Health Services Research Policy, 10(1), 45-53.

8.- Asokan, I., Rabadia, S. V., \& Yang, E. H. "The COVID-19 Pandemic and its Impact on the Cardio-Oncology Population. Current oncology reports". [PubMed Base de datos en Internet], (2020). 22(6), 60. [Acceso el 17 de agosto de

https://doi.org/10.1007/s11912-020-

00945-4 Disponible en: https://www.ncbi.nlm.nih.gov/pmc/article s/PMC7253235/

9.- Lin, L., Yan, L., Liu, Y., Yuan, F., Li, H., $\& \mathrm{Ni}$, J. "Incidence and death in 29 cancer groups in 2017 and trend analysis from 1990 to 2017 from the Global Burden of Disease Study. Journal of hematology \& oncology", [PubMed Base de datos de ineternet]2019. 12(1), 96. [Acceso el 18 de agosto de 2020] https://doi.org/10.1186/s13045-019-

0783-9 Disponible en: https://www.ncbi.nlm.nih.gov/pmc/article s/PMC6740016/

10.- Hui, D., Paiva, CE, Del Fabbro, et al. "Pronóstico en cáncer avanzado: actualización y direcciones para futuras investigaciones. Atención de apoyo en el cáncer: revista oficial de la Asociación Multinacional de Atención de Apoyo en el Cáncer". [PubMed Base de datos en Irnet] 2019. 27 (6), 1973-1984. https://doi.org/10.1007/s00520-019-

04727-y Disponible en: https://www.ncbi.nlm.nih.gov/pmc/article s/PMC6500464/

11.- GBD 2017 Colorectal Cancer Collaborators (2019). "The global, regional, and national burden of colorectal cancer and its attributable risk factors in 195 countries and territories, 1990-2017: a systematic analysis for the Global Burden of Disease Study 2017. The lancet. Gastroenterology \& hepatology", [PubMed base de datos en internet] 2017 4(12), 913-933. https://doi.org/10.1016/S24681253(19)30345-0 [Acceso el 18 de agosto de 2020] Disponible en: https://www.ncbi.nlm.nih.gov/pmc/article s/PMC7026697/

12.- Budny, A., Grochowski, C., et al. "Obesity as a tumour development triggering factor. Annals of agricultural and environmental medicine: AAEM". [Internet]2019, 26(1), 13-23. 
https://doi.org/10.26444/aaem/100664

Recuperado de:

http://www.aaem.pl/Obesity-as-a-

Tumor-Development-Triggering-

Factor, 100664,0,2.html

13.- Shah, S. C., Kayamba, V., Peek, R. M., Jr, \& Heimburger, D." Cancer Control in Low- and Middle-Income Countries: Is It Time to Consider Screening. Journal of global oncology" [PubMed Base de datos en internet] 2019. 5, 1-8. https://doi.org/10.1200/JGO.18.00200

Disponible en: https://www.ncbi.nlm.nih.gov/pmc/article s/PMC6452918/

14.- Martínez-Poveda, B., Torres-Vargas, J. A., Ocaña, M., et al, "The Mediterranean Diet, a Rich Source of Angiopreventive Compounds in Cancer. Nutrients" [PubMed base de datos en internet] 2019, 11(9), 2036. [Acceso el 18 de agosto de 2020] https://doi.org/10.3390/un11092036

Disponible en: https://www.ncbi.nlm.nih.gov/pmc/article s/PMC6769787/

15.- Hayes, S. C., Newton, R. U., Spence, R. R., \& Galvão, D. A. "The Exercise and Sports Science Australia position statement: Exercise medicine in cancer management. Journal of science and medicine in sport" [base de datos de Internet] 2019, 22(11), 1175-1199. https://doi.org/10.1016/j.jsams.2019.05. 003 Disponible en: https://pubmed.ncbi.nlm.nih.gov/312779 21/

16.- Seebacher, N. A., Stacy, A. E., Porter, G. M., \& Merlot, A. M. "Clinical development of targeted and immune based anti-cancer therapies. Journal of experimental \& clinical cancer research: CR" [PubMed base de datos en internet] 2019, 38(1), 156 [acceso el 18 de agosto de

https://doi.org/10.1186/s13046-019-

1094-2 Disponible en: https://www.ncbi.nlm.nih.gov/pmc/article s/PMC6460662/

17.- Patel, C., Brotherton, J. M., et al "The impact of 10 years of human papillomavirus (HPV) vaccination in Australia: what additional disease burden will a nonavalent vaccine prevent. Euro surveillance: bulletin Europeen sur les maladies comunicables $=$ European omunicable disease bulletin". [PubMed base de datos en internet] 2018, 23(41), 1700737. [Acceso el 18 de agosto de 2020]https://doi.org/10.2807/15607917.ES.2018.23.41.1700737 Disponible en:

https://www.ncbi.nlm.nih.gov/pmc/article s/PMC6194907/

18.- Ock, M., Choi, W. J., \& Jo, M. W. "Trend analysis of major cancer statistics according to sex and severity levels in Korea. PloS one" [ PubMed base de datos en internet] 2018, 13(9), e0203110. [Acceso el 18 de agosto de 2020] https://doi.org/10.1371/journal.pone.020 3110 Disponible en: https://www.ncbi.nlm.nih.gov/pmc/article s/PMC6136735/

19.- India State-Level Disease Burden Initiative Cancer Collaborators. "The burden of cancers and their variations across the states of India: The Global Burden of Disease Study 1990-2016. The Lancet. Oncology", [PubMed base de datos en internet]2018 19(10), 1289-1306. [Acceso el 18 de agosto de 2020] https://doi.org/10.1016/S1470-

2045(18)30447-9 Disponible en: https://www.ncbi.nlm.nih.gov/pmc/article s/PMC6167407/

20.- Bellanger, M., Zeinomar, N., Tehranifar, P., \& Terry, M. B. "Are Global Breast Cancer Incidence and Mortality Patterns Related to Country-Specific Economic Development and Prevention Strategies? Journal of global oncology", [PubMed Base de datos en internet] 2018 4, 1-16. [Acceso el 18 de agosto de 2020] https://doi.org/10.1200/JGO.17.00207

Disponible en: https://www.ncbi.nlm.nih.gov/pmc/article s/PMC6223528/

21.- Tu, H., Wen, C. P., et al "Cancer risk associated with chronic diseases and disease markers: prospective cohort study." [PubMed base de datos en internet].2018, BMJ (Clinical research ed.), 360, k134. [Acceso el 18 de agosto de

https://doi.org/10.1136/bmj.k134

Disponible 2020]

en: https://www.ncbi.nlm.nih.gov/pmc/article s/PMC5791146/

22.- Akinyemiju, T., Wiener, H. y Pisu, M., "Factores de riesgo relacionados con el cáncer e incidencia de cánceres importantes por raza, género y región; análisis del estudio de salud y dieta NIHAARP. Cáncer de BMC", [PubMed base de datos en internet] 201717 (1), 597. [Acceso el 18 de agosto de 2020] 
https://doi.org/10.1186/s12885-017-

3557-1 Disponible en: https://www.ncbi.nlm.nih.gov/pmc/article s/PMC5577755/

23.- Elidrissi E.M., Ouarzane, M., Boulouiz, R., \& Bellaoui, M. "Cancer incidence in eastern Morocco: cancer patterns and incidence trends", [PubMed base de datos en internet] 2017. 2005-2012. BMC cáncer, 17(1), 587. [Acceso el 18 de agosto de 2020] https://doi.org/10.1186/s12885-017-

3597-6 Disponible en: https://pubmed.ncbi.nlm.nih.gov/288513 24/

24.- Ribatti D. "The concept of immune surveillance against tumors. The first theories. Oncotarget" [PubMed base de datos en internet]2017, 8(4), 7175-7180. [Acceso el 18 de agosto de 2020]https://doi.org/10.18632/oncotarge t.12739 Disponible en: https://www.ncbi.nlm.nih.gov/pmc/article s/PMC5351698/

25.- Cree, I. A., Uttley, L., et al, "Early Cancer Detection Consortium, The evidence base for circulating tumour DNA blood-based biomarkers for the early detection of cancer: a systematic mapping review." [PubMed base de datos en Internet] 2017. BMC un, 17(1), 697. [Acceso el 18 de agosto de 2020] https://doi.org/10.1186/s12885-017-

3693-7 Disponible en: https://www.ncbi.nlm.nih.gov/pmc/article s/PMC5654013/

26.- Roquette, R., Painho, M., \& Nunes, B. "Spatial epidemiology of cancer: a review of data sources, methods and risk factors". [Internet] 2017. Geospatial health, 12(1), 504. [Acceso el 18 de agosto de 2020] https://doi.org/10.4081/gh.2017.504

Disponible

en: https://pubmed.ncbi.nlm.nih.gov/285554 68/

27.- Ireland, M. J., March, S., CrawfordWilliams, F., et al "A systematic review of geographical differences in management and outcomes for colorectal cancer in Australia." [PubMed base de datos en internet] 2017. BMC cáncer, 17(1), 95. [Acceso el 18 de agosto de 2020] https://doi.org/10.1186/s12885-017-

3067-1 Disponible en: https://www.ncbi.nlm.nih.gov/pmc/article s/PMC5290650/

28.- Organización Panamericana de la Salud (OPS). "La OMS describe los pasos para salvar 7 millones de vidas amenazadas por el cáncer". [Internet] 2020. Disponible en: https://www.paho.org/hq/index.php?opti on $=$ com_content\&view $=$ article\&id $=15708$ :who-outlines-steps-to-save-7-millionlives-from-cáncer\&Itemid $=1926 \&$ ime $=$ es 29.- Organización Mundial de la Salud (OMS), "Estrategia mundial sobre régimen alimentario, actividad física y salud," [internet]2004 ISBN: $924359222 \mathrm{X}$, Clasificación LC/NLM: QT 180, [Acceso el 17 de agosto de 2020] Disponible en: https://www.who.int/dietphysicalactivity/ strategy/eb11344/strategy_spanish_web. pdf?ua $=1$ 
Tabla 3, ANEXO 1: Artículos

incluidos en la revisión.

\begin{tabular}{|c|c|c|c|}
\hline Autor y Año & Objetivo & Tipo de Estudio & Elementos útiles \\
\hline $\begin{array}{l}\text { (Asokan, I., } \\
\text { Rabadia, S. V.,' } \\
\text { \& Yang, E. H., } \\
\text { 2020). (8) }\end{array}$ & $\begin{array}{l}\text { Conocer la relación } \\
\text { entre pacientes con } \\
\text { enfermedades } \\
\text { cardiovasculares y/o } \\
\text { oncológicas } \\
\text { estrategias y } \\
\text { atenuar el riesgo de } \\
\text { tratamiento, manejo y } \\
\text { vigilancia en esta } \\
\text { población y el Covid-19. }\end{array}$ & $\begin{array}{lr}\text { Revisión de la } \\
\text { literatura. Explora } \\
\text { los factores de } \\
\text { riesgo, } \\
\text { diagnósticos } \\
\text { opciones y } \\
\text { tratamiento que } \\
\text { afectan a los } \\
\text { pacientes cardio- } \\
\text { oncológicos con } \\
\text { COVID-19. }\end{array}$ & $\begin{array}{l}\text { Los pacientes con cáncer } \\
\text { tienen un mayor riesgo de } \\
\text { sufrir una lesión cardíaca. } \\
\text { Aquellos que se someten } \\
\text { a una terapia contra el } \\
\text { cáncer tienen en teoría } \\
\text { una mayor susceptibilidad } \\
\text { a la infección. La } \\
\text { hipertensión, } \\
\text { enfermedades } \\
\text { cardiovasculares, las } \\
\text { diabetes y el cáncer se } \\
\text { han asociado con una } \\
\text { infección por covID-19 } \\
\text { más grave y con peores } \\
\text { resultados. }\end{array}$ \\
\hline $\begin{array}{ll}\text { (Lin, } & \text { L.et al, } \\
2019) & (9)\end{array}$ & $\begin{array}{l}\text { Determinar las tasas de } \\
\text { incidencia y mortalidad } \\
\text { por edad de } 29 \text { grupos } \\
\text { de cáncer. }\end{array}$ & $\begin{array}{l}\text { Análisis de } \\
\text { tendencia para } \\
\text { los principales } \\
\text { tipos de cáncer en } \\
\text { función de sexo, } \\
\text { edad, región y } \\
\text { país. }\end{array}$ & $\begin{array}{l}\text { Según datos de } 2017 \text { las } \\
\text { tasas de mortalidad más } \\
\text { altas son para: cáncer de } \\
\text { tráquea, bronquios y } \\
\text { pulmón (TBL), cáncer de } \\
\text { colon y recto, cáncer de } \\
\text { estómago y cáncer de } \\
\text { hígado, mama, páncreas, } \\
\text { esófago, próstata, otras } \\
\text { neoplasias malignas y } \\
\text { leucemia. El cáncer de } \\
\text { TBL se considera el más } \\
\text { mortal con un } 19,9 \% \text { del } \\
\text { total. En hombres la } \\
\text { morbilidad y mortalidad } \\
\text { es mayor que en mujeres. } \\
\text { Los mayores de } 50 \text { años } \\
\text { tienen un alto riesgo de } \\
\text { desarrollarlo. Australia y } \\
\text { nueva Zelanda registran } \\
\text { las cifras más altas. }\end{array}$ \\
\hline $\begin{array}{l}\text { (Hui, D., et al, } \\
\text { 2019) (10) }\end{array}$ & $\begin{array}{l}\text { Proporcionar una } \\
\text { actualización sobre el } \\
\text { pronóstico en pacientes } \\
\text { con cáncer avanzado. }\end{array}$ & $\begin{array}{l}\text { Revisión sobre el } \\
\text { pronóstico en } \\
\text { pacientes con } \\
\text { cáncer avanzado } \\
\text { para una } \\
\text { predicción precisa } \\
\text { y proponer } \\
\text { direcciones } \\
\text { futuras para la } \\
\text { investigación del } \\
\text { pronóstico. }\end{array}$ & $\begin{array}{l}\text { Desde } 2005 \text { un Grupo de } \\
\text { Trabajo de la Red de } \\
\text { Investigación de la } \\
\text { Asociación Europea de } \\
\text { Cuidados Paliativos } \\
\text { identificó } \\
\text { recomendaciones } \\
\text { basadas en la evidencia } \\
\text { sobre el pronóstico en el } \\
\text { cáncer avanzado para } \\
\text { mejorar la precisión de la } \\
\text { predicción de la } \\
\text { supervivencia, pero aún } \\
\text { ha de progresar y } \\
\text { desarrollar futuras } \\
\text { investigaciones. }\end{array}$ \\
\hline
\end{tabular}



Colorectal Cancer Collaborators, 2019). (11)
(GBD $2017 \mid$ Determinar el estado sobre la incidencia, la mortalidad y la discapacidad causadas por el cáncer colorrectal en 195 países y territorios entre 1990 y 2017.
Análisis de datos de registro vital, y registro de cáncer para generar estimaciones de incidencia,

muerte y años de vida ajustados por discapacidad (AVAD) del cáncer colorrectal a nivel mundial, regional nacional.
De 1990 a 2017 la tasa de incidencia aumento a nivel mundial, siendo el $36^{a}$ causa principal de carga de enfermedad a nivel mundial para 2017 y la $4^{\circ}$ causa principal de muerte detrás del de pulmón, hígado y estómago. Las tasas de incidencia estandarizadas por edad más altas en 2017 se registraron en Australasia, Asia Pacífico de ingresos altos $y$ América del Norte de ingresos altos, aunque la tendencia es a disminuir debido a la introducción de pruebas de detección y eliminación de pólipos precancerosos durante las colonoscopias, y las tasas de mortalidad estandarizadas por edad más altas se encontraron en Europa central, Europa oriental y sur de América Latina.

Se ha demostrado la relación entre el exceso de grasa corporal y las neoplasias. La obesidad está asociada con muchos cánceres, como el de mama, colorrectal, hígado, pulmón, etc....

Registro alto de

(Shah, S. C., $\quad$ Determinar el éxito en
Kayamba, V., programas de detección Peek, R. M., Jr, temprana del cáncer. \& Heimburger, D., 2019).

(13)

\begin{tabular}{l} 
\\
\hline (Martínez- \\
Poveda, B., et \\
al, 2019) (14)
\end{tabular}

Conocer aquellos
alimentos que presentan actividad anti angiogénica y su posible actuación como agente angio preventivo. sistemática de la literatura científica respecto.

Revisión

analiza compromete éxito de programas control del cáncer (detección temprana) países ingresos medios y bajos.

Revisión
sistemática sobre los compuestos bioactivos naturales de los alimentos de la dieta mediterránea. mortalidad en los países de ingresos bajos $y$ medios en personas menores de 65 años. Impulsar la prevención y tratamiento es fundamental para el desarrollo sostenible de dichos países.

Existe evidencia científica
de los beneficios que ciertos alimentos aportan a la salud imprescindible en su papel preventivo y terapéutico, (como el aceite de oliva, frutas y verduras que poseen fisetina un flavonol bioactivo con efectos antiinflamatorios, antidiabéticos antioxidantes, 


\begin{tabular}{|c|c|c|c|}
\hline & & & $\begin{array}{lr}\text { antitumorales, } & \text { anti } \\
\text { invasores, } & \text { anti } \\
\text { angiogénicos, } & \text { neuro } \\
\text { protectores } & \text { y } \\
\text { cardioprotectores). } & \\
\end{array}$ \\
\hline $\begin{array}{l}\text { (Hayes, S. C., } \\
\text { Newton, R. U., } \\
\text { Spence, R. R., } \\
\text { \& Galvão, D. } \\
\text { A., 2019) (15) }\end{array}$ & $\begin{array}{l}\text { Evaluación del papel del } \\
\text { ejercicio en la salud del } \\
\text { individuo y en el } \\
\text { tratamiento del cáncer. }\end{array}$ & $\begin{array}{lr}\begin{array}{l}\text { Informe } \\
\text { emplea }\end{array} & \begin{array}{r}\text { que } \\
\text { evidencia } \\
\text { científica y }\end{array} \\
\begin{array}{l}\text { experiencia } \\
\text { clínica sobre }\end{array} & \text { la } \\
\text { prescripción } & \text { de } \\
\text { ejercicios } & \\
\text { específicos } & \text { para } \\
\text { el cáncer. } & \end{array}$ & $\begin{array}{l}\text { La implementación del } \\
\text { ejercicio conlleva la } \\
\text { reducción de la la } \\
\text { morbilidad, mejora la } \\
\text { funcionalidad y calidad de } \\
\text { vida, mejora la } \\
\text { supervivencia, viabilidad } \\
\text { y reduce el riesgo de } \\
\text { daño. }\end{array}$ \\
\hline $\begin{array}{l}\text { (Seebacher, } \\
\text { N. A., Stacy, A. } \\
\text { E., Porter, G. } \\
\text { M., \& Merlot, } \\
\text { A. M., 2019) } \\
\text { (16) }\end{array}$ & $\begin{array}{l}\text { Conocer el éxito de las } \\
\text { terapias dirigidas contra } \\
\text { el cáncer. }\end{array}$ & $\begin{array}{l}\text { Revisión } \\
\text { bibliográfica para } \\
\text { determinar el } \\
\text { desarrollo clínico, } \\
\text { éxitos y desafíos } \\
\text { que enfrentan las } \\
\text { terapias dirigidas } \\
\text { contra el cáncer. }\end{array}$ & $\begin{array}{l}\text { Las terapias dirigidas } \\
\text { contra el cáncer están } \\
\text { suponiendo } \\
\text { revolución ya que la } \\
\text { especificidad, menor } \\
\text { toxicidad y capacidad de } \\
\text { activación del sistema } \\
\text { inmunológico de estos } \\
\text { agentes han sido muy } \\
\text { prometedores para el } \\
\text { tratamiento del cáncer. }\end{array}$ \\
\hline $\begin{array}{l}\text { (Patel, C., et al } \\
\text { 2018) (17) }\end{array}$ & $\begin{array}{l}\text { Conocer el impacto del } \\
\text { programa nacional de } \\
\text { vacunación contra el } \\
\text { VPH en Australia. }\end{array}$ & $\begin{array}{l}\text { Revisión } \\
\text { bibliográfica no } \\
\text { sistemática de } \\
\text { estudios que } \\
\text { midieron la carga } \\
\text { de enfermedad e } \\
\text { infección } \\
\text { asociadas al VPH } \\
\text { en Australia antes } \\
\text { y después de la } \\
\text { introducción de la } \\
\text { vacuna. } \\
\end{array}$ & $\begin{array}{l}\text { Australia fue de los } \\
\text { primeros países en } \\
\text { financiar e implementar la } \\
\text { vacunación, teniendo un } \\
\text { impacto demostrable en } \\
\text { las enfermedades } \\
\text { relacionadas con el VPH. } \\
\text { Esta podría reducir la } \\
\text { carga de cáncer asociado } \\
\text { al VPH. }\end{array}$ \\
\hline $\begin{array}{l}\text { (Ock, M., Choi, } \\
\text { W. J., \& Jo, M. } \\
\text { W., } \\
\text { (18) 2018) }\end{array}$ & $\begin{array}{l}\text { Conocer la tendencia y } \\
\text { evolución de las tasas } \\
\text { de cáncer en Corea y. }\end{array}$ & $\begin{array}{l}\text { Análisis de las } \\
\text { tendencias en las } \\
\text { principales } \\
\text { estadísticas de } \\
\text { cáncer según } \\
\text { sexo y gravedad } \\
\text { en Corea desde } \\
2006 \text { a } 2013 \text {. }\end{array}$ & $\begin{array}{l}\text { Mediante los datos de } \\
\text { Corea Central Datos del } \\
\text { registro de cáncer se } \\
\text { estimó la tasa de } \\
\text { supervivencia de hasta } 8 \\
\text { años, se observa una } \\
\text { desaceleración y una } \\
\text { disminución del número } \\
\text { de casos. }\end{array}$ \\
\hline $\begin{array}{l}\text { (India State- } \\
\text { Level Disease } \\
\text { Burden } \\
\text { Initiative } \\
\text { Cancer } \\
\text { Collaborators, } \\
\text { 2018).(19) }\end{array}$ & $\begin{array}{lr}\begin{array}{l}\text { Conocer la estimación } \\
\text { de la }\end{array} & \text { mortalidad, } \\
\text { incidencia, prevalencia } \\
\text { y años de }\end{array}$ & Meta análisis. & $\begin{array}{l}\text { Se observa un incremento } \\
\text { de } 1990 \text { a } 2016 \text { de un } \\
90,9 \% \text {, la tasa bruta de } \\
\text { incidencia aumentó un } \\
28,2 \% \text { así como un } \\
\text { registro elevado de } \\
\text { muertes por cáncer } \\
\text { (estómago, } \\
\text { pulmón, faringe, colon, } \\
\text { recto, leucemia y cuello). } \\
\text { Factores de riesgo: } \\
\text { consumo de tabaco y } \\
\text { alcohol y riesgos } \\
\text { dietéticos. }\end{array}$ \\
\hline
\end{tabular}


(Bellanger, M., Conocer la variación et al, 2018) entre incidencia $y$ (20) mortalidad por cáncer de mama.
Análisis descriptivo que compara tasas de incidencia mortalidad.

\section{Estudio}

(Tu, H., et al, Evaluar las asociaciones 2018) (21)

independientes y
principales

enfermedades crónicas

y los marcadores de enfermedades con riesgo de cáncer y explorar el beneficio de la actividad física para reducirlo.

\begin{tabular}{l}
\hline (Akinyemiju, \\
T., Wiener, H. \\
y Pisu, M., \\
2017). (22)
\end{tabular}

Determinar

disparidad racial en relación a la incidencia de cáncer atribuido a factores de riesgo como obesidad, tabaquismo, actividad física y dieta

\begin{tabular}{l}
\hline Elidrissi \\
Errahhali M, \\
2017) (23)
\end{tabular}

Conocer los casos
registrados en el Centro Regional de Oncología Hassan II (ROC) conjuntas de las cohorte

prospectivo de 405.878

participantes.
Existe una incidencia elevada en niveles de ingresos altos en todas las edades y en países más pobres soportan la mayor carga de mortalidad en menores de 50 años.

de La enfermedad crónica es un factor de riesgo, contribuye en más de $1 / 5$ parte del riesgo de incidencia y más de $1 / 3$ de muerte.

La actividad física se asocia a una reducción del $40 \%$ en el riesgo de cáncer asociado con enfermedades crónicas.

Estudio de Se observa una baja

Cohorte adherencia de la prospectiva de incidencia del cáncer 566.398 adultos sugiriendo oportunidades (blancos y para desarrollar afroamericanos). $\quad$ estrategias de prevención

Se registraron un total de descriptivo $\quad 7.872$ casos desde 2005 a retrospectivo de 2012, (5220 mujeres y pacientes 2652 hombres).

diagnosticados de El cáncer de mama ocupa cáncer de entre 2005 y 2012. el primer puesto. La mayoría son desempleados y sin seguro médico procedentes de zonas urbanas y rurales.

\begin{tabular}{|c|c|c|c|}
\hline $\begin{array}{l}\text { (Ribatti D., } \\
\text { 2017). (24) }\end{array}$ & $\begin{array}{l}\text { Conocer las diversas } \\
\text { teorías sobre vigilancia } \\
\text { inmunitaria. }\end{array}$ & $\begin{array}{l}\text { Artículo de } \\
\text { revisión de la } \\
\text { literatura. }\end{array}$ & $\begin{array}{l}\text { Existe un nuevo enfoque } \\
\text { llamado inmunoeditación } \\
\text { compuesto de tres fases: } \\
\text { eliminación de células NK, } \\
\text { CD4 + y CD8 +, equilibrio } \\
\text { entre las células } \\
\text { inmunitarias y tumorales. }\end{array}$ \\
\hline $\begin{array}{l}\text { (Cree, I. A., et } \\
\text { al, 2017) (25) }\end{array}$ & $\begin{array}{l}\text { Determinar la literatura } \\
\text { existente sobre } \\
\text { biomarcadores } \\
\text { sanguíneos para la } \\
\text { detección de cáncer en } \\
\text { humanos. }\end{array}$ & $\begin{array}{l}\text { Revisión de } \\
\text { mapeo mediante } \\
\text { búsqueda en } \\
\text { bases de datos. }\end{array}$ & $\begin{array}{l}\text { Se identifican } \\
\text { consideraciones } \\
\text { preanalíticas, analíticas y } \\
\text { posanalíticas que deben } \\
\text { tratarse antes de que los } \\
\text { biomarcadores entren en } \\
\text { la práctica clínica. Se } \\
\text { requiere de estudios más } \\
\text { grandes. } \\
\text { El ctDNA puede ser una } \\
\text { opción viable para la } \\
\text { detección temprana pero } \\
\text { no todos son detectables } \\
\text { con los métodos actuales. }\end{array}$ \\
\hline $\begin{array}{l}\text { (Roquette, R., } \\
\text { Painho, M., \& } \\
\text { Nunes, } \\
\text { 2017). (26) }\end{array}$ & $\begin{array}{ll}\text { Determinar } & \text { la } \\
\text { importancia de la } \\
\text { epidemiología para } & \text { el } \\
\text { diseño de políticas de } & \end{array}$ & $\begin{array}{l}\text { Revisión } r \text { y } \\
\text { discusión de la } \\
\text { literatura entre } \\
1979 \text { y } 2015 \text {. }\end{array}$ & $\begin{array}{l}\text { Análisis de } 180 \text { artículos } \\
\text { de } 63 \text { revistas. } \\
\text { Se observa un abordaje } \\
\text { sustancial en la última }\end{array}$ \\
\hline
\end{tabular}




\begin{tabular}{|c|c|c|c|}
\hline & $\begin{array}{l}\text { salud pública destinadas } \\
\text { a minimizar el impacto } \\
\text { de las enfermedades } \\
\text { crónicas en poblaciones } \\
\text { específicas. }\end{array}$ & & $\begin{array}{l}\text { década sobre la } \\
\text { epidemiologia espacial del } \\
\text { cáncer. Adquiriendo } \\
\text { relevancia para futuras } \\
\text { investigaciones y la falta } \\
\text { de estudios. }\end{array}$ \\
\hline $\begin{array}{l}\text { (Ireland, M. J., } \\
\text { et al, 2017) } \\
(27)\end{array}$ & $\begin{array}{l}\text { Identificación de las } \\
\text { disparidades } \\
\text { geográficas en la } \\
\text { supervivencia rel del } \\
\text { Cáncer colorrectal, el } \\
\text { tratamiento clínico y los } \\
\text { resultados } \\
\text { psicosociales. }\end{array}$ & $\begin{array}{l}\text { Revisión } \\
\text { mediante las } \\
\text { pautas PRISMA y } \\
\text { realizando una } \\
\text { revisión en } 7 \\
\text { bases de datos. }\end{array}$ & $\begin{array}{l}\text { Australia y Nueva Zelanda } \\
\text { registran una tasa muy } \\
\text { elevada de cáncer } \\
\text { colorrectal suponiendo } \\
\text { una carga sanitaria, } \\
\text { económica y rocial } \\
\text { importante. El área } \\
\text { regional de residencia } \\
\text { juega un papel crucial. }\end{array}$ \\
\hline $\begin{array}{l}\text { (Quante A., et } \\
\text { al 2016) (5) }\end{array}$ & $\begin{array}{l}\text { Determinar la carga } \\
\text { futura de cáncer en } \\
\text { Alemania. }\end{array}$ & $\begin{array}{l}\text { Recopilación de } \\
\text { datos y análisis } \\
\text { del Instituto } \\
\text { Robert Kock. }\end{array}$ & $\begin{array}{l}\text { Mediante los métodos } \\
\text { aplicados por Rahib y } \\
\text { colegas prevé que la } \\
\text { incidencia de la mayoría } \\
\text { de los canceres aumente } \\
\text { hasta } 2030 \text { superando el } \\
\text { de próstata al de mama y } \\
\text { convirtiéndose en la } \\
\text { neoplasia más frecuente. } \\
\text { El cáncer de pulmón y de } \\
\text { páncreas superara al } \\
\text { colorrectal y ocupara el } \\
\text { tercer lugar entre los } \\
\text { canceres más comunes. } \\
\text { El cáncer de mama será el } \\
\text { más común en mujeres } \\
\text { con un aumento } \\
\text { constante en el número } \\
\text { de casos en las próximas } \\
\text { décadas. }\end{array}$ \\
\hline
\end{tabular}

